\title{
An examination of the distinction between nouns and verbs: Associations with two different kinds of motion
}

\author{
ALAN W. KERSTEN \\ Indiana University, Bloomington, Indiana
}

\begin{abstract}
Four experiments provide evidence that people are biased to associate particular types of motion with nouns and different types of motion with verbs. Novel nouns and verbs were related to two types of motion: (1) path, or the direction of motion of one character with respect to the other character, and (2) movement orientation, or the direction a character was facing as it moved. Subjects associated verbs more strongly with path than with movement orientation. In contrast, they associated nouns more strongly with movement orientation than with path. Movement orientation was associated with both object categories and verbs, inconsistent with a complete division of labor between these two types of categories. These results are consistent, however, with the notion that people are biased to associate verbs with relations between objects, whereas they are biased to associate object categories with motions defined with respect to the object carrying out those motions.
\end{abstract}

In order to learn the meaning of a word, people must learn the relation of that word to relevant aspects of the environment. It would be very difficult, however, to determine which aspects of the environment were relevant, if one did not have ways of narrowing down the number of possible hypotheses for the meaning of a word. One way of narrowing down the possible meanings of a word is to use syntactic cues. L. R. Gleitman, H. Gleitman, Landau, and Wanner (1987) refer to this use of syntax as syntactic bootstrapping. For example, Naigles (1990) showed that, when children were presented with a novel verb, they focused on different aspects of an event, depending on whether the verb was presented in a transitive or an intransitive sentence frame. In particular, when children heard the sentence "The duck is gorping the bunny," they focused on what the duck was doing to the bunny. In contrast, when they heard the sentence "The duck and the bunny are gorping," they focused on actions the two characters were carrying out independently.

Perhaps even more basic than the distinction between different verb types is the distinction between nouns and verbs. Just as the number of arguments presented along with a verb provides a syntactic cue for the meaning of that

Experiments 1 and 3 were part of a dissertation conducted in partial fulfillment of the requirements for the Doctor of Philosophy degree at the Georgia Institute of Technology. I gratefully acknowledge the contributions of my dissertation committee: Dorrit Billman, Susan Bovair, Kurt Eiselt, Tim Salthouse, and Tony Simon. I also thank Julie Earles and Rob Goldstone for thoughtful comments on earlier versions of the paper. Please address correspondence to A. W. Kersten, Department of Psychology, Florida Atlantic University, 777 Glades Rd., Boca Raton, FL 33431-0991 (e-mail: akersten@fau.edu). Examples of the events used in these experiments are available over the World Wide Web at http://www. psy.fau.edu/chez/awk

-Accepted by previous editor, Geoffrey R. Loftus verb, there are numerous syntactic cues that can assist in determining whether a novel word is a noun or a verb. Maratsos and Chalkley (1980) proposed that the grammatical categories of noun and verb are formed on the basis of such syntactic cues. For example, nouns often end in $-s$ and follow the or $a$, whereas verbs often end in -ed or -ing and follow is. This theory explains how people can maintain separate grammatical categories for nouns and verbs, even though there is considerable overlap in the meanings they convey (e.g., an attack vs. to attack).

Work as early as that of Brown (1957), however, has shown that children go beyond these syntactic cues and entertain different hypotheses for the meaning of a word, depending on whether it is a noun or a verb. For example, when children learned a novel noun (e.g., $a$ sib), they usually extended this label only to the same object. In contrast, when children learned a novel verb (e.g., sibbing), they usually extended this label to actions. According to Brown, "children develop firm, and temporarily reliable, notions about the semantics of nouns and verbs. These notions may stay with them as adults even though they retain only a probabilistic truth" (p. 2). Indeed, there is evidence that even adults focus on different aspects of an event, depending on whether they are learning nouns or verbs, as demonstrated both from studies of observational learning (Kersten, 1998) and learning from context (Nagy \& Gentner, 1990).

It thus seems that, although there is overlap in the meanings of nouns and verbs, people may have very different core notions as to the types of information that nouns and verbs convey. It is not clear, however, exactly what these core notions are. The textbook definition of a noun is that it is a word for an object, such as a person, place, or thing. Verbs, in turn, are thought of as the primary vehicles for conveying information about the motions of such objects. 
Recent research, however, has provided evidence that motions are associated with both nouns and verbs (Kersten, 1998; Kersten \& Billman, 1995).

This research has demonstrated that people are biased to attend to different kinds of motion when learning nouns than when learning verbs. Kersten (1998) demonstrated that people are biased to associate nouns with intrinsic motion, or motion defined with respect to the object carrying out that motion. For example, a noun may describe not only the appearance of the limbs and body of an animal but also the ways that those limbs move relative to the body. Thus, dog may refer not only to a creature with four legs and a tail but also to the wagging motion of the tail. In contrast, people are biased to associate verbs with extrinsic motion, or the motion of one object with respect to a second object. For example, in the sentence The dog chased the squirrel, the verb chased describes the path of the dog with respect to the squirrel. The function of these learning biases may be to create a division of labor between nouns and verbs in the description of motion, with nouns and verbs conveying different types of motion. Thus, both nouns and verbs are necessary to provide a detailed description of motion. I will refer to this theory as the division of labor hypothesis. This theory stands in opposition to the traditional notion that relational terms such as verbs are responsible for the description of motion and that nouns merely serve as labels for objects.

The division of labor hypothesis was tested in Kersten (1998) by presenting adult subjects with animated events involving two characters. One character, the agent, moved throughout each event, while a second character, the patient, remained stationary, serving as a reference point for the agent's motion. Each event was accompanied by a sentence with a novel noun and verb. Each noun and verb were always accompanied by a particular intrinsic motion (i.e., the leg motion of the agent), as well as a particular extrinsic motion (i.e., the path of the agent with respect to the patient). In the absence of learning biases, nouns and verbs would show the same pattern of associations with these two types of motion. The subjects, however, associated nouns more strongly with intrinsic than with extrinsic motion, whereas they associated verbs more strongly with extrinsic than with intrinsic motion.

The events in Kersten (1998) were relatively simple, in that only one character moved in each event. The present study was designed to test whether these biases extend to more complex events in which more than one character is in motion. In addition, many real-world events involve motions that are less clear examples of intrinsic or extrinsic motion than those used in Kersten. The present study examined associations involving an attribute that is similar in some respects to both intrinsic and extrinsic motion. Thus, the present research was designed to offer a broader characterization of the kinds of motion that people tend to associate with nouns and verbs.

The present experiments compared the association of two different kinds of motion with nouns and verbs. One kind of motion, patient path, involved the path of the pa- tient with respect to the agent. For example, the sentence The cheetah chased the gazelle implicates a motion of the gazelle away from the cheetah. Patient path is, thus, an example of extrinsic motion. The second kind of motion involved the motion of the agent with respect to its orientation, or the direction it was facing. For example, most animals move head first, but others are sidewinders, and some (e.g., people) are capable of moving backwards. This kind of motion will be referred to as movement orientation, to distinguish it from the static orientation of an object (e.g., facing toward vs. away from the viewer). Movement orientation is similar to path in that it involves the motion of an object as a whole rather than the motions of its parts. Because movement orientation can be defined without reference to any other objects, however, it is an example of intrinsic motion. For example, backing up by the agent can be defined as motion opposite to the direction faced by the agent's head and, thus, can be differentiated from other values of movement orientation by reference only to body parts of the agent.

Because movement orientation involves intrinsic motion, the division of labor hypothesis predicted that nouns would be more strongly associated with movement orientation than with patient path, an extrinsic motion attribute. In contrast, it was predicted that verbs would be more strongly associated with patient path than with movement orientation.

\section{The Association of Movement Orientation With Nouns and Verbs}

Evidence regarding the association of movement orientation with verbs can be found in a prior experiment by Kersten (1998). In one condition of this experiment, movement orientation was diagnostic of verbs, whereas, in the other condition, movement orientation varied randomly and agent path was diagnostic. The strength of associations between verbs and movement orientations could, thus, be compared to associations between verbs and an extrinsic motion attribute (i.e., agent path). This comparison revealed that subjects associated verbs more strongly with agent path than with movement orientation. Thus, although movement orientation involved the motion of the agent as a whole, just as did agent path, subjects were less likely to associate verbs with movement orientation, perhaps because it involved intrinsic motion.

Movement orientation was never diagnostic of nouns in the experiments of Kersten (1998). Thus, this work provides no evidence regarding the association of movement orientation with nouns. Other work, however, suggests that movement orientation does play a role in object categories and, thus, may also be associated with nouns.

Kersten and Billman (1997) found evidence suggesting that movement orientation plays a role in object categories. They presented subjects with an unsupervised event category learning task, in which no category labels were provided. Events varied on a number of attributes. Some were global event attributes, such as state change--that is, the effect of a causal interaction between two charac- 
ters on the appearance of one of the characters. Other attributes were more intrinsic to individual objects, such as the leg motion of an object. The subjects had difficulty learning correlations between state change and leg motion. Kersten and Billman (1997) suggested that the subjects associated state change with event categories, whereas they associated leg motion with object categories. As a result, the subjects had difficulty relating leg motion and state change. For some subjects, however, movement orientation was related to both leg motion and state change. These subjects were better at associating leg motion and state change with movement orientation than with each other. Thus, the subjects inferred relations between state change and leg motion only after each was associated with movement orientation. Kersten and Billman (1997) interpreted this finding as indicating that movement orientation was associated with both object and event categories.

Movement orientation involves the representation of the motion of an object with respect to the orientation of that object. Although there has been little research directly examining the association of movement orientation with nouns, there is evidence that canonical orientations are associated with object categories. For example, the canonical orientation for a human is upright, facing forward. Evidence for a role of orientation in object categories comes from research on a neurological impairment called hemineglect. This impairment involves a difficulty in attending to information appearing on the contralesional (typically left) side of space (see Bisiach \& Vallar, 1988, for a review). For example, hemineglect patients often omit details on the left side of a picture when asked to copy it and may even fail to bathe or apply cosmetics to the left sides of their bodies.

What constitutes left has traditionally been defined with respect to the patient, such that items in the left visual field or items to the left of the midline of the patient may be difficult to attend to. Recent evidence, however, suggests that hemineglect can also operate with respect to the canonical orientations of perceived objects (Behrmann \& Moscovitch, 1994; Caramazza \& Hillis, 1990; Driver \& Halligan, 1991). In particular, when objects are rotated from their canonical orientations, neglect is seen for areas of the objects that would have fallen on the left if they were in their canonical orientations, even when these areas now fall on the right visual field. Behrmann and Moscovitch offer the example of a neglect patient who may have difficulty orienting to the number 9 on a clock face, even when the clock is rotated $180^{\circ}$. This evidence suggests that hemineglect can operate at the level of individual object representations. These representations include information about an object's canonical orientation, and left and right are characterized with respect to this orientation.

There is evidence that the canonical orientation of an object influences the interpretation of spatial relations involving that object. For example, prepositions such as in front of and behind are often ambiguous when used with nouns such as person or house that have canonical orientations (Jackendoff, 1987; Talmy, 1983). Behind usually means on the other side of some object or location. Behind the house, however, is often considered to refer to a location to the back side of the house -that is, to the direction faced by the back door. Thus, the sentence John is behind the house is ambiguous if it is said while one is in the backyard, as John could be thought of as being either to the far side of the house (i.e., in the front yard) or to the back of the house (i.e., in the backyard). This suggests that object concepts such as house have canonical fronts and backs and that spatial relations are interpreted with respect to this canonical orientation.

Carlson-Radvansky and Irwin (1993) provided further evidence for a role of object orientation in the interpretation of spatial relations. They presented subjects with scenes involving two objects (e.g., a fly and a donkey) and asked them to evaluate descriptions of the scenes, such as The fly is above the donkey. In some trials, the reference object (i.e., the donkey) was in its canonical orientation. There was thus only one region that could be considered to be above the donkey-namely, the region above the donkey's back. In other trials, called noncanonical trials, the donkey was rotated $90^{\circ}$, as if climbing a steep hill. Thus, in the noncanonical trials, there were two potential placements of the fly, relative to the donkey, that could be considered to be above. One was above the donkey in terms of an gravitational (extrinsic) frame of reference - that is, farther above the ground than the donkey's front legs and head. The second was above in terms of an object-centered (intrinsic) frame of reference - that is, above the donkey's back after taking its orientation into account. The subjects rated descriptions involving above as being more acceptable when the fly was in either of these locations than when it was not above the reference object in terms of any frame of reference. Furthermore, ratings were higher in the canonical trials, in which the fly was above in terms of both frames of reference, than in the noncanonical trials in which the fly was only above the reference object in terms of an extrinsic frame of reference. These findings suggest that the intrinsic reference frame specified by an object's canonical orientation influences people's interpretations of spatial relations involving that object.

Evidence for influences of an object's canonical orientation on the interpretation of static spatial relations suggests that an object's orientation may also play a role in the interpretation of the motions of that object. There are similarities between the role of orientation in the representation of static spatial relations and in the representation of motion, as is depicted in Figure 1. The first panel of Figure 1 represents a static spatial relation and could be described by the sentence The crumb is to the left of the bug. The second panel represents motion and could be described by the sentence The bug moves to its left. Each of the above sentences is correct only with respect to the canonical orientation of the bug. If, on the other 


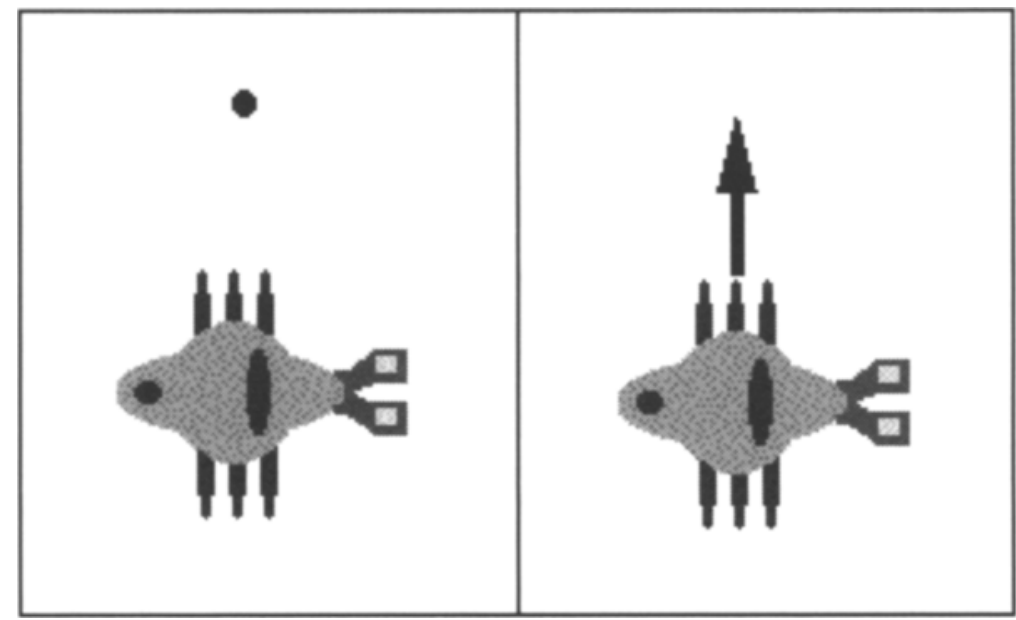

Figure 1. Comparison of the static spatial relation to the left of the bug and motion to its left.

hand, one adopted an extrinsic reference frame (i.e., one that is viewer or environment centered), the crumb would be described as being above the bug.

The difference between the two sentences represented in Figure 1 is that the first sentence describes the location of the crumb with respect to the bug, whereas the second sentence describes the direction of motion (and thus the future location) of the bug itself. Thus, given the evidence that objects are associated with canonical orientations and that these orientations are used in the interpretation of spatial relations, it is likely that an object's motion is also interpreted with respect to its canonical orientation. If this movement orientation were consistent for a particular category of objects, this information would likely become associated with that category. For example, if the type of bug shown in Figure 1 were always shown moving to its left, that would suggest that being a sidewinder was a characteristic of that kind of bug. Thus, object categories may convey information about movement orientation.

\section{The Association of Patient Motion With Nouns and Verbs}

Extrinsic motion involves the motion of an agent, defined with respect to a reference object. Thus, one way to manipulate extrinsic motion is to vary the path of the agent relative to a stationary patient, as was done in the experiments of Kersten (1998) and Kersten and Billman (1995). A second way to vary extrinsic motion, however, is to vary the path of the patient, rather than requiring it to remain stationary throughout the event. Motion of the patient is clearly implicated by many verbs, such as collide and chase. As can be seen in Figure 2, the same agent motion can be described by either of these verbs. The distinction between these verbs involves whether the patient moves toward or away from the agent.

The implication of patient motion by many verbs is consistent with the hypothesis that people tend to associate verbs with extrinsic motion. Because this motion is carried by a character other than the agent, it allows a strong test of the extrinsic motion bias hypothesis. In particular, this extrinsic motion involving the patient can be compared with a more intrinsic motion attribute carried by the agent (i.e., movement orientation). The extrinsic motion bias hypothesis predicts that people will associate verbs more strongly with the patient path than with the movement orientation of the agent, even if the patient is never explicitly mentioned in the accompanying sentence. In contrast, if one relied only on the syntax of the accompanying sentence, one would expect movement orientation to be easier to associate with verbs, because it was carried by the only character mentioned in the accompanying sentence.

It is possible that patient motion is also associated with nouns. Nelson (1983) proposed that children's earliest object concepts center on the roles played by objects within events. For example, a child's first concept of dog may include objects that play the role of chaser in events involving other objects, such as cats, cars, squirrels, and so forth. According to this theory, only later does intrinsic infor-

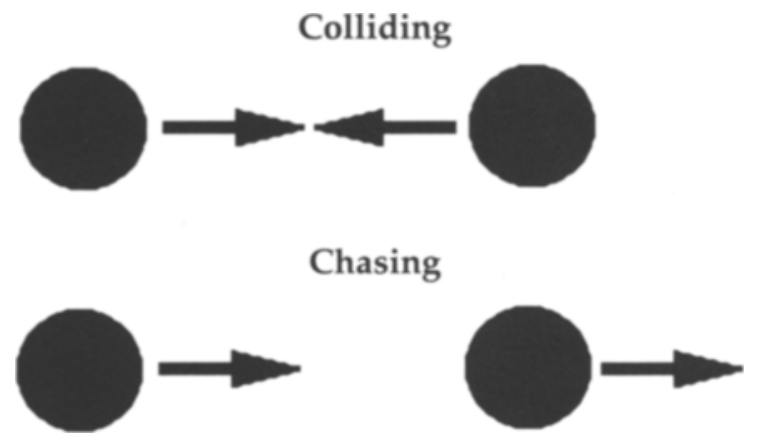

Figure 2. Comparison of chasing and colliding. The agent in each event is depicted on the left, whereas the patient is on the right. Note that the motion of the agent is the same in both cases, whereas the patient's motion varies. 
mation, such as the form of an object, start to embellish these earlier object concepts. The role of an agent within an event depends not only on the actions of the agent itself but also on the actions of the other objects with which it interacts (e.g., a squirrel must run away in order for a dog to chase it). Thus, one could interpret this theory as suggesting that the patient path should play a role in the formation of object concepts for agents within events. Because the patient path involves extrinsic motion, however, the division of labor hypothesis predicts that the patient path should be less strongly associated with nouns than are intrinsic motion attributes such as movement orientation.

\section{EXPERIMENT 1}

This experiment was designed to test for associations of nouns and verbs with movement orientation and patient path. These two attributes were equally related to nouns and verbs during the learning phase of this experiment. The subjects were then tested for their knowledge of these relations. The subjects were predicted to associate nouns more strongly with movement orientation than with patient path, as a result of an intrinsic motion bias. In contrast, the subjects were predicted to associate verbs more strongly with patient path than with movement orientation, because of an extrinsic motion bias. If the intrinsic and extrinsic motion biases do not apply to movement orientation and patient path, however, one would expect a similar pattern of association of each type of motion with nouns and verbs.

\section{Method}

\section{Subjects}

Thirty-two undergraduates at the Georgia Institute of Technology received course credit for participation in this experiment.

\section{Stimuli}

All events. MacroMind Director 2.0 was used to generate events that were displayed on Macintosh II computers. Figure 3 depicts an example of such an event. Each event involved the motions of two characters. One of the characters, the agent, was composed of three attributes: head, body, and legs. Figure 4 depicts the four values of each of these attributes. The other character, the patient, did not have interchangeable parts. Patient appearance was, instead, a unitary attribute, with four possible values (see Figure 4). There were also four possible values of the environment in which the event took place. The environment consisted of two identical line drawings that appeared in two unoccupied corners of the screen.

There were also a number of attributes related to the motions of the two characters (see Figure 5). One attribute was agent path, or the direction(s) of motion of the agent with respect to the patient. A second attribute was the leg motion of the agent. Each subject saw all four values of path and leg motion. The third attribute was the movement orientation of the agent, or whether the agent moved in the direction it faced, backed up, or moved sideways to the left or right. Although movement orientation had four possible values, each subject saw only two of these values. These two values were selected at random, with the constraint that no subject saw only leftward motion and rightward motion (agents were nearly symmetrical around the front-back axis, and, thus, the subjects may not have expected a distinction between leftward and rightward motion to be important).
A fourth attribute, patient path, had only two possible values, with the patient moving either toward or away from the agent.

A black screen faded out to reveal the starting positions of the two characters at the beginning of each event. The agent started at a location randomly chosen from a set of points near the center of the screen. The patient started at a location randomly chosen from eight possible directions relative to the agent, either N, S, E, W, NE, $\mathrm{NW}, \mathrm{SE}$, or SW. An event continued until the agent came in contact with the patient or had traveled an equivalent distance away from the patient. The starting locations of the two characters were related to patient path, with the agent and patient starting closer together when the patient moved away from the agent. The patient moved at half the speed of the agent so that the agent could catch up with the patient when they moved in the same direction.

Learning events. There were 80 learning events. A sentence presented in a female voice accompanied each learning event. The sentence began as soon as the characters started in motion and ended shortly before the end of the event. Each sentence was composed of a novel noun, preceded by the, and a novel verb, preceded by is and followed by -ing. Four different nouns (i.e., racha, doovil, zeebee, and taigo) and four different verbs (i.e., spogging, morping, yimming, and wunking) were heard by each subject. A button labeled Next Event appeared after each learning event, allowing the subject to continue.

The values of several attributes were correlated with nouns and verbs during learning. Figure 6 depicts the correlations seen by an example subject. These correlations are only offered as an example because the actual pairings of attribute values varied randomly across subjects. As shown in Figure 6, each noun was always accompanied by an agent with a particular type of legs as well as a particular leg motion. Each verb was always accompanied by a particular agent path. For example, the noun taigo labeled an agent with two legs on each side that moved up and down along the length of the agent's body, whereas the verb morping labeled motion of the agent directly toward the patient. If a subject only wanted to distinguish the four nouns and verbs, these attributes would have been sufficient. Two additional attributes, however, were correlated with both nouns and verbs. One attribute was movement orientation, and the other was patient path.

For each subject, movement orientation and patient path had two values. Each of these values was correlated with two nouns and two verbs. For example, moving backwards in Figure 6 is correlated not only with the nouns zeebee and doovil, but also with the verbs morping and spogging. The path of the patient away from the agent is correlated with the same two nouns and two verbs. Thus, there was a shared superordinate structure for nouns and verbs, with two nouns and two verbs correlated with one movement orientation and one patient path, and two other nouns and two other verbs correlated with the other movement orientation and patient path. All of the remaining, uncorrelated attributes (i.e., agent head, agent body, patient appearance, environment) took one of four values, chosen at random in each event. This random variation was included so that a particular sentence did not always occur with exactly the same event, which may have encouraged subjects simply to associate sentences with whole events.

Word association test events. Twenty-four trials tested for associations involving individual nouns and verbs. Each trial involved a forced choice between two events. The first event in each test trial was presented along with a question about an individual noun (e.g., Is this a doovil?) or verb (e.g.. Is this spogging?). The same question accompanied the second event. The presentation of each ques tion started at the beginning of an event and finished about halfway through the event. The subjects were asked to decide which event was a better example of the accompanying noun or verb. One event in each trial was a perfect example of the noun or verb, whereas the other involved one attribute value that was inconsistent with the 

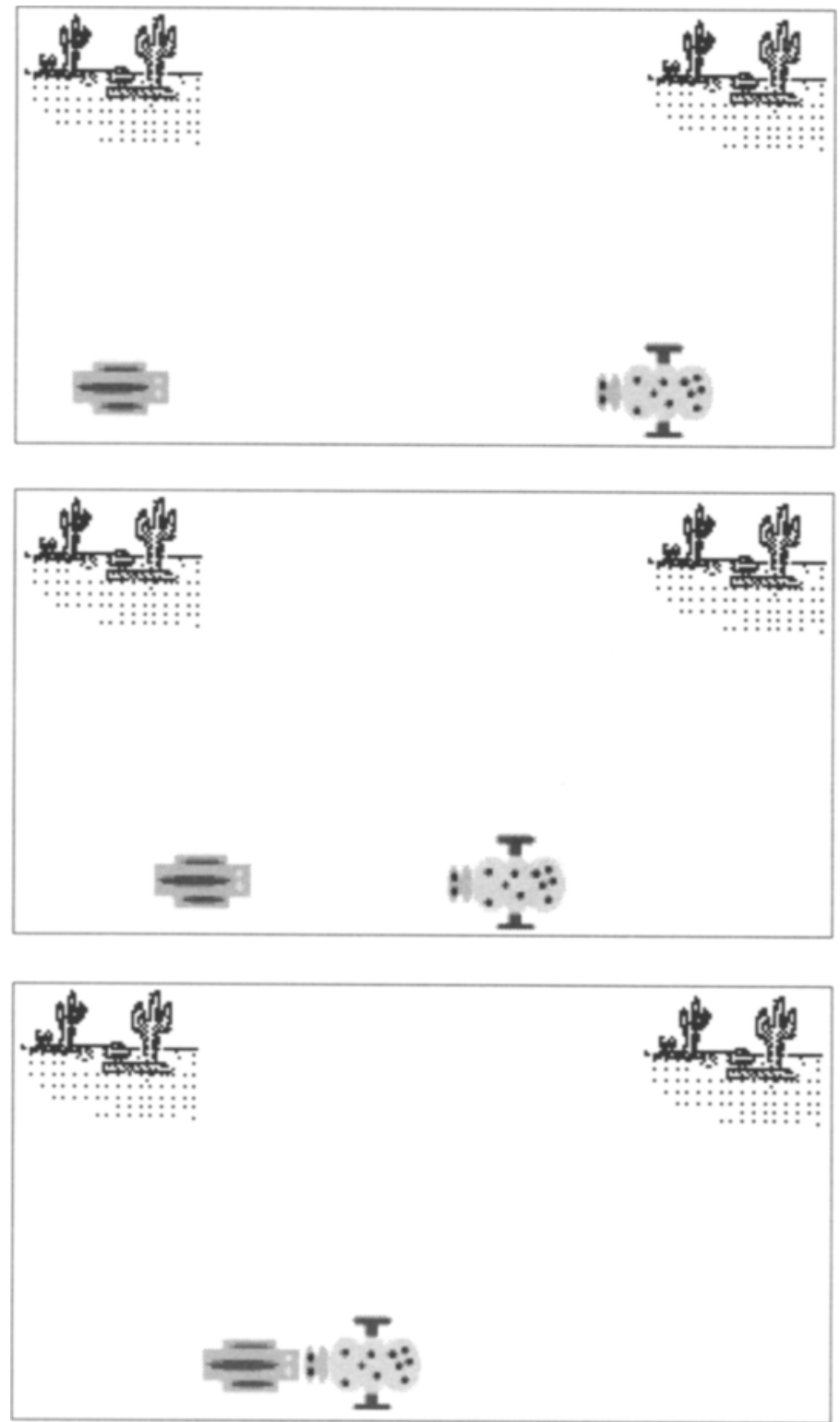

Figure 3. Three frames from an example event. The speckled agent moves toward the striped patient. The patient approaches the agent throughout the event. The actual stimuli were displayed in 16 colors. $A$ sentence such as the zeebee is morping was heard while the event was taking place.

word. The strength of the association between a noun or verb and a particular attribute value was indicated by a subject's ability to choose the entirely correct event as the better example of the word.

Eight trials tested for associations involving movement orientation, and eight tested for associations involving patient path. Half of these trials tested for knowledge of nouns, whereas half tested for knowledge of verbs. In addition, four trials tested for associations between nouns and the values of agent legs, whereas four tested for associations between verbs and agent paths. All attributes that were not being tested in a given trial took on the values that they had during learning. Thus, if an attribute was related to nouns or verbs during learning, it was given a value consistent with the noun or verb in the test trial. If an attribute varied randomly during learning, it continued to vary randomly during testing.

Mismatch test events. A second test evaluated whether the subjects considered it to be more important for the values of a particular attribute to be consistent with nouns or with verbs. Each of 16 trials involved a combination of noun and verb that had not been presented during learning. As can be seen in Figure 6, during learning each noun was used in the same sentence with only two of the four verbs. Similarly, each verb was used together with only two of the four nouns. In each mismatch trial, a noun was used together with a verb it had never been paired with during learning. For example, in Figure 6 the verb morping could be paired with racha or taigo. 
Agent Head
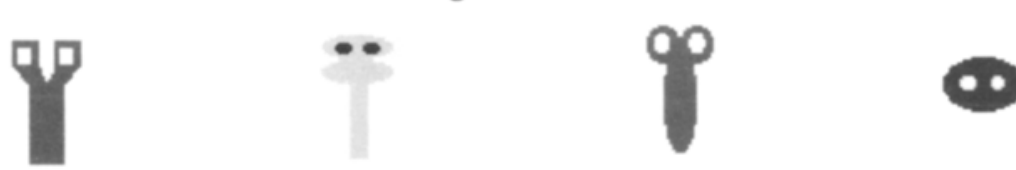

Agent Body
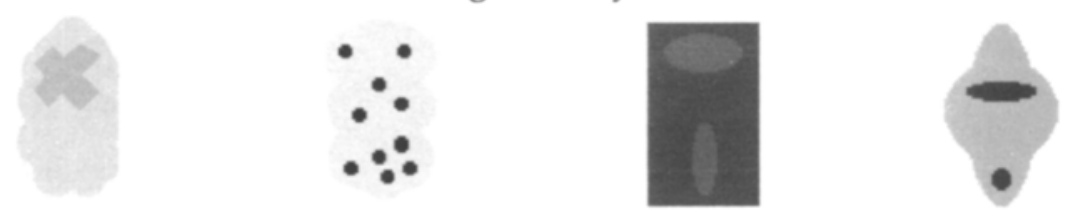

Agent Legs

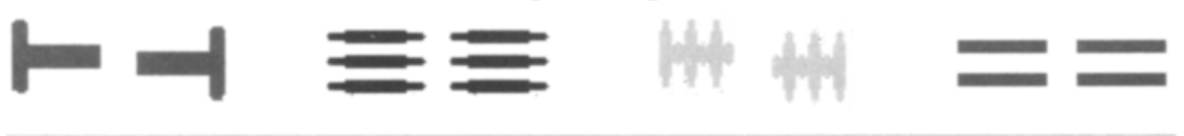

Patient Appearance
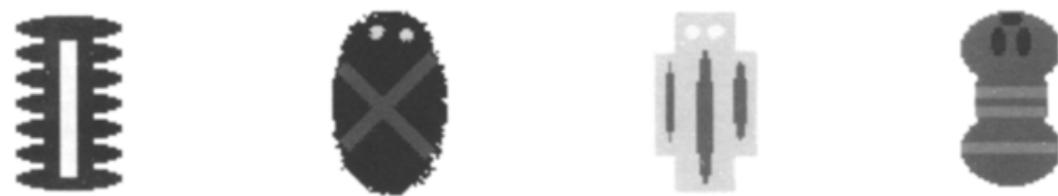

Environment
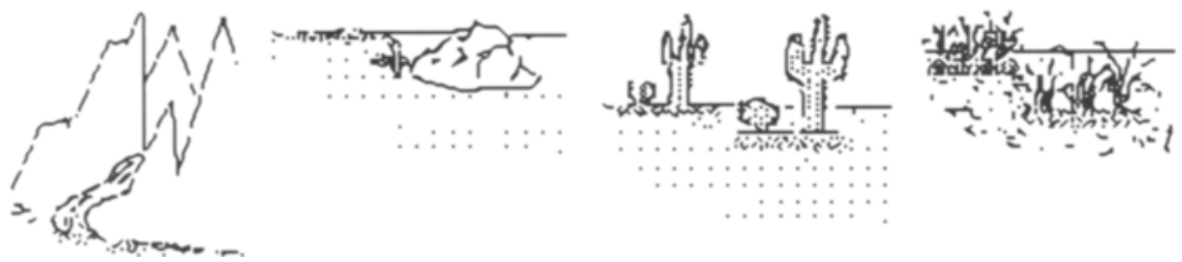

Figure 4. The four possible values of each of the static attributes in the events.

As with the word association trials, each mismatch trial involved two events. In one event (the orientation $=$ verb, path $=$ verb event, or OVPV), the values of movement orientation and patient path were consistent with the verb in the sentence but inconsistent with the noun. In the other event - either orientation $=$ noun, path $=$ verb $($ ONPV $)$, or orientation $=$ verb, path $=$ noun $($ OVPN $)$-one of these two attributes took a value that was consistent with the noun but inconsistent with the verb, whereas the other took a value consistent with the verb. At the end of each trial, the subjects were asked to choose which of the two events was a better example of the sentence. If a subject chose the event in which one attribute was consistent with the noun but inconsistent with the verb, this would provide evidence that the attribute was more strongly associated with the noun than with the verb. If. instead, the subject chose the event in which the attribute was consistent with the verb but inconsistent with the noun, this would indicate that the attribute was more strongly associated with the verb. Movement orientation varied on eight trials (i.c.. OVPV vs. ONPV), whereas patient path varied in the other eight (i.e.. OVPV vs. OVPN). In every cvent, the values of agent legs and leg motion were consistent with the noun, whereas the value of agent path was consistent with the verb. All other attributes were assigned values in the same way as they were during learning.

As an example, a subject who was assigned the schema in Figure 6 would have been presented with a novel sentence, The racha is morping. The agent in this trial would have had three legs on each side of its body, and these legs would have angled forward and back as the agent moved. The path of the agent would have been directly toward the patient. In one event in this trial (OVPV), the agent's movement orientation would have been backwards, and the patient path would have been away from the agent, both consistent with the verb morping. In the other event in this trial, either movement orientation would have been forward (ONPV) or patient path would have been toward the agent (OVPN), depending on whether movement orientation or patient path varied in that trial.

\section{Design}

The primary dependent measure in this experiment was accuracy at choosing the correct events in the word association test trials. The 


\section{Agent Path}
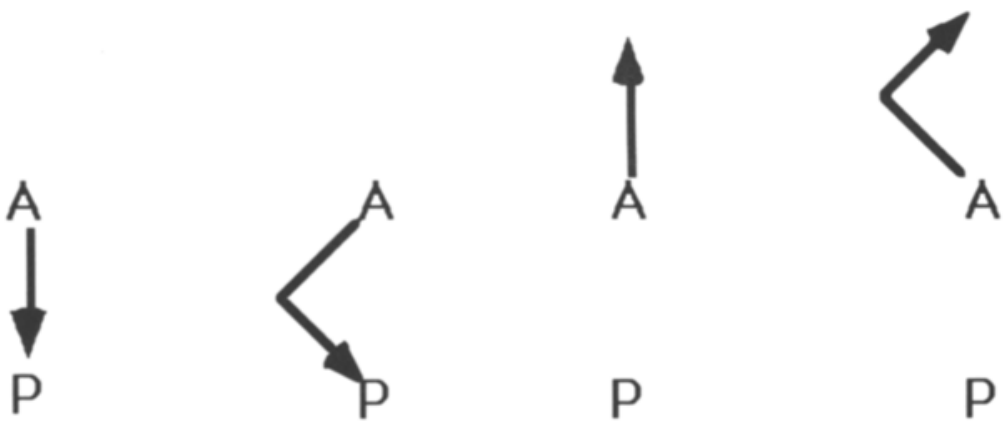

\section{Leg Motion}
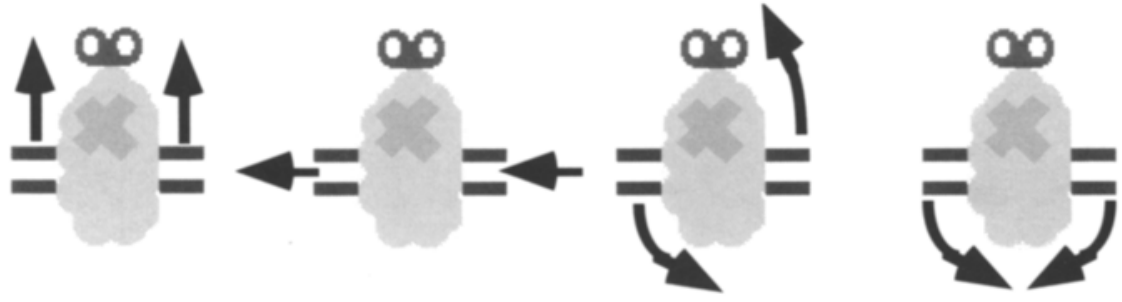

\section{Orientation}
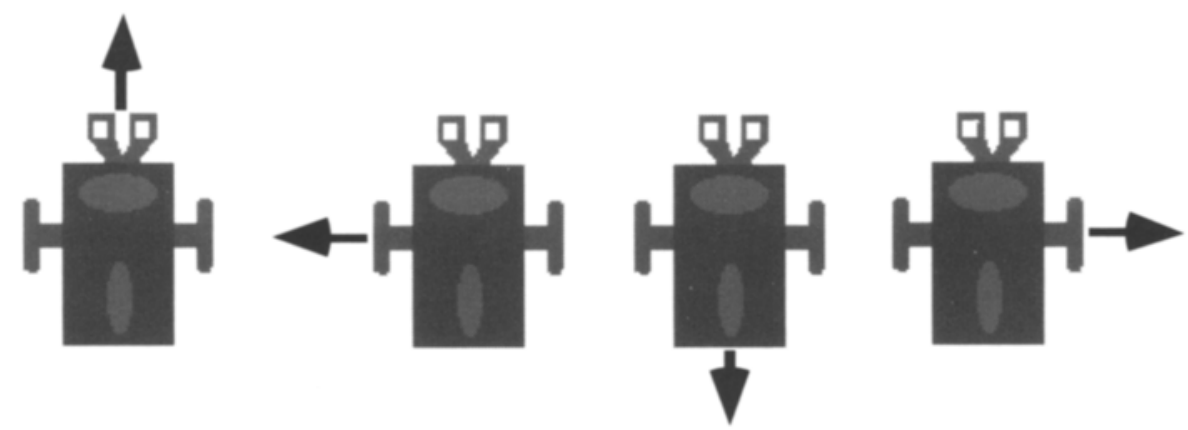

\section{Patient Path}

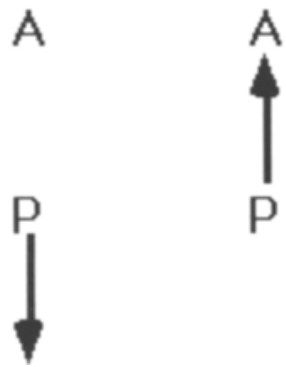

Figure 5. The possible values of each of the motion attributes in the events. Arrows represent the direction of motion. $A$, agent; $P$, patient.

independent variables were the part of speech of the word accompanying each test trial (noun vs. verb) and the attribute that varied over that trial (movement orientation vs. patient path). A second dependent measure was the percentage of choices that were consistent with the verb rather than with the noun in the mismatch trials. The independent variable for this dependent measure was the attribute that varied over each trial (movement orientation vs. patient path).

\section{Procedure}

Sessions were self-paced and lasted approximately $45 \mathrm{~min}$. Instructions were presented on the computer. The subjects were instructed that they would see a number of events depicting life on another planet and that they were to learn the meanings of words accompanying those events. Eighty learning events were then presented. At the end of learning, the subjects were instructed that they 


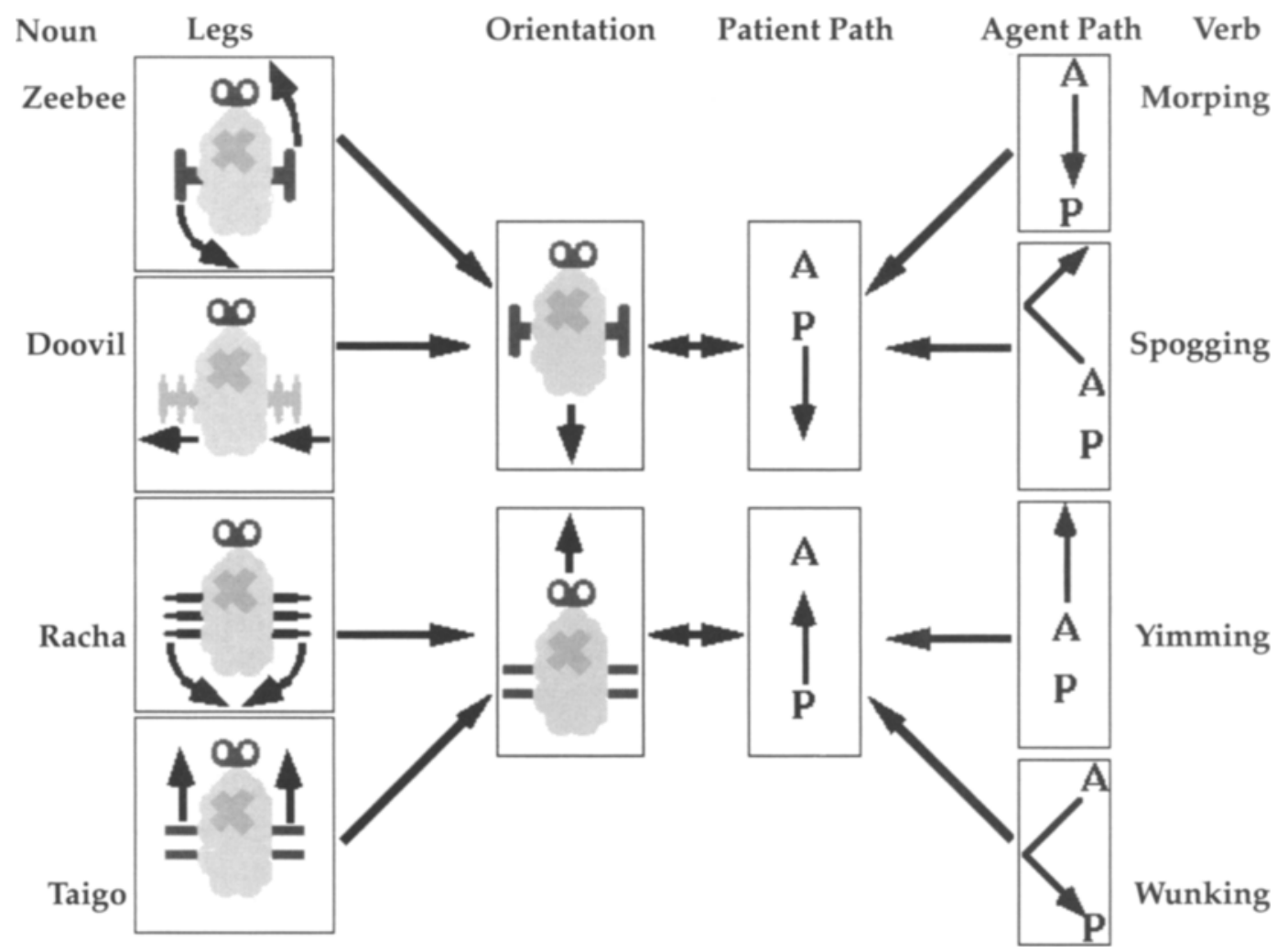

Figure 6. Correlations seen by an example subject in Experiment 1. A, agent; P, patient.

would be tested on their knowledge of the nouns and verbs presented during learning. They were then presented with 24 test trials, each of which involved two events. After the first event in each trial, the subject clicked on a button labeled Next Event to see the second event in the trial. After the second event, subjects pressed one of three buttons. The Repeat button allowed the subjects to view the two events again. The other two buttons were labeled First Event and Second Event. Subjects clicked on the button corresponding to the event that they thought was a better example of the accompanying word. After the word association trials, the subjects were instructed that they would be asked to interpret sentences involving combinations of nouns and verbs that had never gone together during learning. They were instructed that there were no right or wrong answers. They were then presented with 16 mismatch trials.

\section{Results}

\section{Word Association Test Events}

The results of the word association test events are depicted in Figure 7. An alpha level of .05 was adopted for all analyses. A 2 (part of speech, noun vs. verb) $\times 2$ (attribute, movement orientation vs. patient path) analysis of variance (ANOVA) was conducted on forced-choice accuracy. This analysis revealed that the subjects were significantly more accurate on relations involving verbs than on relations involving nouns $\left[F(1,31)=10.99, M S_{\mathrm{e}}=513.48, p<.01\right]$. There was no significant main effect of the attribute being tested $[F(1,31)<1]$. There was, however, a significant interaction of part of speech and attribute $[F(1,31)=$ 17.61, $\left.M S_{\mathrm{e}}=443.55, p<.001\right]$. The subjects associated nouns significantly more strongly with movement orientation than with patient path $[t(31)=2.03, p<.05$, onetailed]. In contrast, the subjects associated verbs significantly more strongly with patient path than with movement orientation $[t(31)=4.03, p<.001$, one-tailed $]$.

Broken down by attribute rather than by part of speech, the subjects associated patient path significantly more

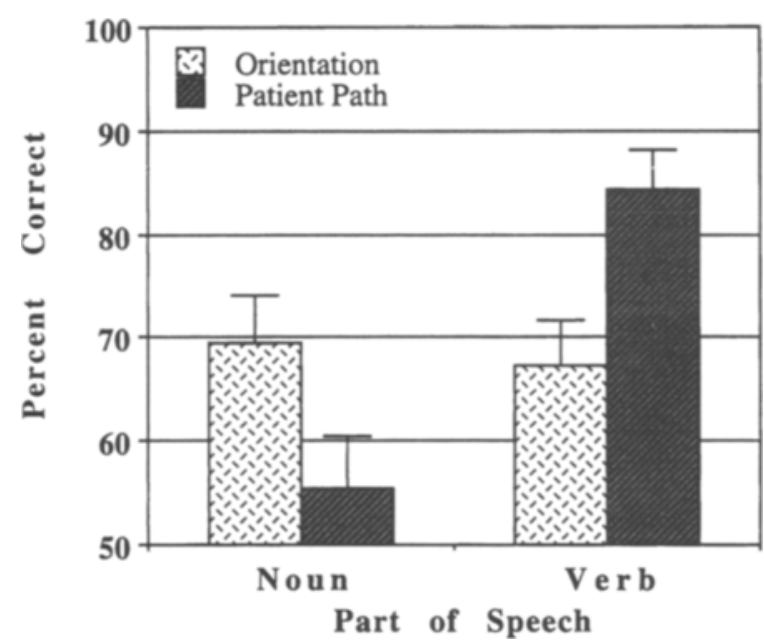

Figure 7. Results of the word association trials in Experiment 1 , in terms of mean percent correct and standard errors. 
strongly with verbs than with nouns $[t(31)=4.95, p<$ .001 , one-tailed]. In fact, the subjects did not perform significantly above chance on associations between patient paths and nouns $(p>.10)$. On trials testing knowledge of movement orientation, there was no significant difference between nouns and verbs $[t(31)=0.46, p>.10]$. This lack of a significant effect was not a result of a floor effect. The subjects performed with above-chance accuracy on relations of movement orientation to both nouns and verbs $(p<.001)$.

Word association trials also tested knowledge of relations involving agent legs and agent path, in order to ensure that the subjects were able to differentiate the four nouns and verbs in terms of these two attributes. The subjects showed considerable knowledge of these relations, averaging $79.7 \%(S D=25.0 \%)$ correct on trials testing knowledge of relations between nouns and the values of agent legs and $93.8 \%(S D=15.6 \%)$ on trials testing knowledge of relations between verbs and agent paths. Thus, because the subjects could differentiate nouns and verbs in terms of these two attributes, they were not compelled by the experimental task to associate nouns and verbs with movement orientation and patient path.

\section{Mismatch Trials}

The results of the mismatch trials are depicted in Figure 8 . On trials in which patient path varied, significantly more than $50 \%$ of the subjects' choices were consistent with the verb and inconsistent with the noun $(p<.001)$. Thus, patient path was more strongly associated with verbs than with nouns. In contrast, on trials in which movement orientation varied, the percentage of choices consistent with the verb was not significantly different from $50 \%$ $(p>.10)$, indicating that the subjects associated orientation equally strongly with nouns and with verbs. This difference in performance between trials testing patient path and trials testing movement orientation was significant $[t(31)=7.91, p<.001]$.

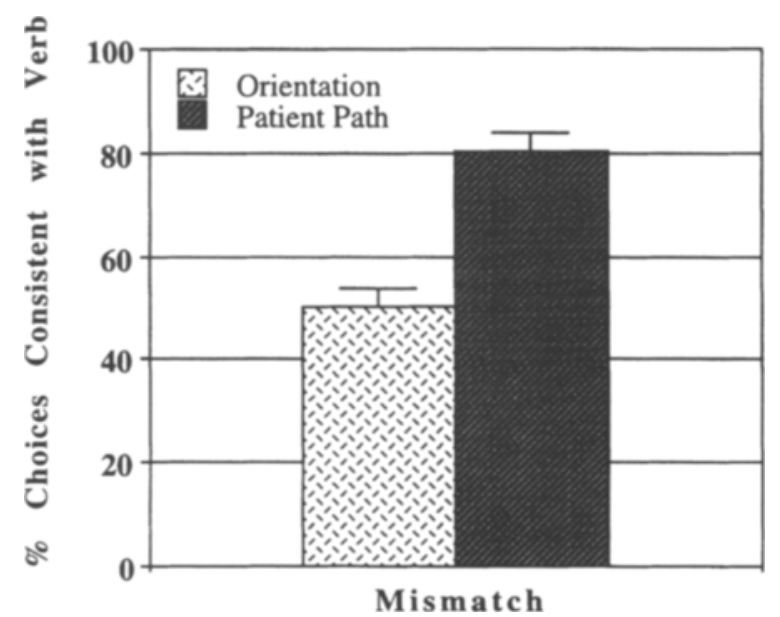

Figure 8. Results of the mismatch trials in Experiment 1.

\section{Discussion}

The results of Experiment 1 revealed a bias to associate verbs more strongly with patient path, an extrinsic motion attribute, than with the movement orientation of the agent, an intrinsic motion attribute. This finding provides strong evidence for an extrinsic motion bias, because only the agent was highlighted by the accompanying sentence. In contrast, the subjects associated nouns more strongly with movement orientation than with patient path.

Although nouns were more strongly associated with movement orientation than with patient path, whereas verbs were more strongly associated with patient path than with movement orientation, overall performance was better with verbs than with nouns. This was a result of patient path being more strongly associated with verbs than with nouns, whereas movement orientation was associated about equally strongly with nouns and with verbs. The finding with patient path was quite consistent with the division of labor hypothesis. The finding that movement orientation was no more strongly associated with nouns than with verbs, however, is inconsistent with the strongest version of the hypothesis that the noun in a sentence is responsible for conveying intrinsic motion. The association of movement orientation with nouns and verbs was explored further in Experiments 3 and 4, whereas Experiment 2 further investigated associations involving patient path.

\section{EXPERIMENT 2}

Experiment 1 revealed that the subjects had difficulty learning relations between patient paths and nouns, whereas they were better at associating patient paths with verbs. This is consistent with the hypothesis that people expect verbs, but not nouns, to convey extrinsic motion. An alternative explanation, however, comes from the fact that nouns held a consistent relation only to patient path and not to agent path. For example, a subject assigned the schema in Figure 6 would have seen the noun zeebee paired with an agent path toward the patient on some events (i.e., when it was morping) and away from the patient on others (i.e., when it was spogging). Thus, the relation of the agent to the patient was different with different instances of the same nouns, perhaps making it difficult to discern the underlying consistent relation between nouns and patient path. In Experiment 2, each noun and verb held a consistent relation to the paths of both the agent and the patient.

An additional reason why the subjects in Experiment 1 may have had difficulty associating nouns with patient paths is that the appearance of the patient varied randomly. Characteristic interactions associated with a particular noun are generally dependent on the other objects involved. For example, cats tend to chase mice, but one should not characterize them as chasers in general, because they more often flee from dogs. The subjects in Experiment $\mathbf{l}$ may have been hesitant to associate a particular role (e.g., chaser) with a noun without regard to 


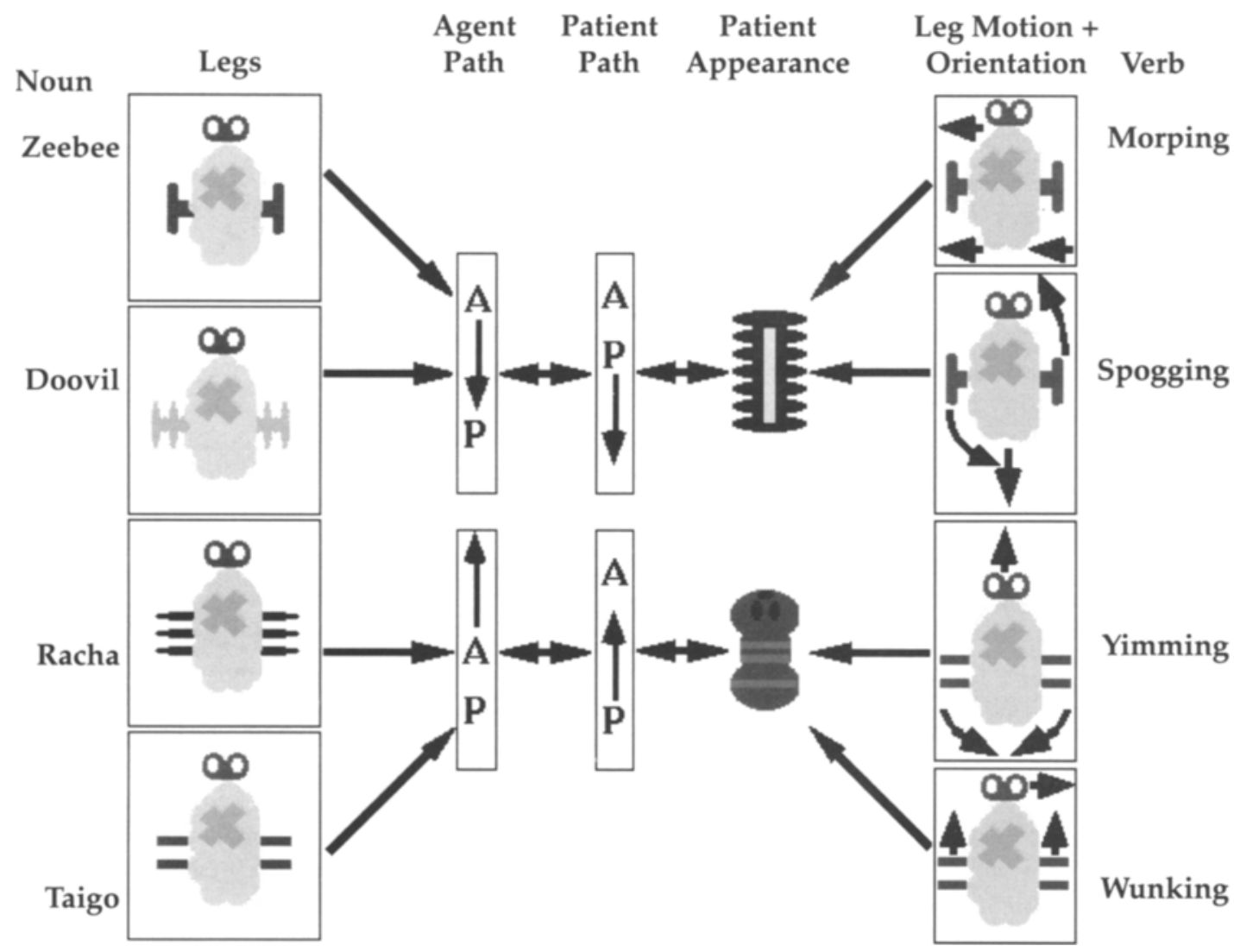

Figure 9. Correlations seen by an example subject in Experiment 2. A, agent; P, patient.

the identity of the other object involved. In Experiment 2, each agent always interacted with the same patient, as defined by patient appearance as well as by patient path (see Figure 9). In addition, transitive sentences (e.g., The racha is morping the diggy) were employed so that patients as well as agents received labels, highlighting the connection between an agent and a particular patient. If variation on agent path and patient appearance were responsible for the weaker associations of patient paths with nouns than with verbs in Experiment 1, one would expect these associations to be equal in Experiment 2. Because both patient path and agent path involve extrinsic motion, however, the division of labor hypothesis predicted that subjects would associate both of these attributes more strongly with verbs than with nouns.

\section{Method}

\section{Subjects}

Thirty-two undergraduates at Indiana University participated in this experiment in partial fulfillment of introductory psychology course requirements.

\section{Stimuli}

Learning events. The learning events of Experiment 2 differed from those of Experiment 1 in that each event was accompanied by a transitive rather than an intransitive sentence. The subject noun of each sentence was related to the value of agent legs, as in Experiment 1 (see Figure 9). The object noun was related to the appearance of the patient. Each noun was also related to a particular agent path and a particular patient path. Unlike Experiment I, the four verbs were differentiated by the movement orientation and leg motion of the agent. This allowed agent path as well as patient path to be equally associated with nouns and verbs, while still allowing subjects to fully distinguish the four verbs.

Word association test events. The subjects were tested four times each on relations of subject nouns to agent path, patient path, and agent legs, whereas they were tested on relations of verbs to agent path, patient path, and movement orientation.

Mismatch test events. Patient path varied on eight trials, whereas agent path varied on eight others.

\section{Design}

The primary dependent measure in this experiment was accuracy at choosing the correct events in the word association trials. The independent variables were the part of speech of the word accompanying each test trial (noun vs. verb) and the attribute that varied over that trial (agent path vs. patient path). A second dependent measure was the percentage of choices that were consistent with the verb rather than with the noun in the mismatch trials. The independent variable for this dependent measure was the attribute that varied over each trial (agent path vs. patient path).

\section{Procedure}

The procedure of Experiment 2 was identical to that of Experiment 1 . 


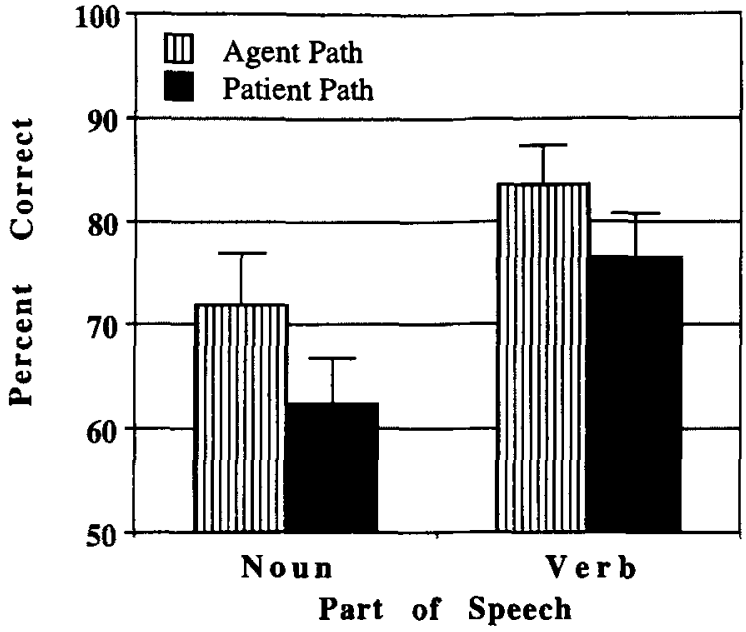

Figure 10. Results of the word association trials in Experiment 2 .

\section{Results \\ Word Association Test Events}

The results of the word association test events are displayed in Figure 10. A 2 (part of speech, noun vs. verb) $\times$ 2 (attribute, patient path vs. agent path) ANOVA was performed on forced-choice accuracy. This analysis revealed that the subjects performed significantly better on tests involving verbs than on tests involving nouns $[F(1,31)=$ $\left.12.42, M S_{\mathrm{e}}=428.27, p<.001\right]$. The subjects associated patient path significantly more strongly with verbs than with nouns $[t(31)=3.48, p<.01]$, although performance was still significantly above chance on relations between nouns and patient paths $(p<.01)$. The subjects also associated agent path significantly more strongly with verbs than with nouns $[t(31)=2.18, p<.05]$, although performance was also significantly above chance on relations between nouns and agent paths $(p<.001)$. The ANOVA also revealed that the subjects were significantly more accurate on tests involving agent path than on tests involving patient path $\left[F(1,31)=7.47, M S_{\mathrm{e}}=\right.$ $288.40, p<.01]$. There were significantly stronger associations to agent path than to patient path for nouns $[t(31)=2.04, p<.05]$, whereas this trend approached significance for verbs $[t(31)=1.79, p<.09]$. There was no evidence for an interaction between part of speech and attribute $[F(1,31)=0.15, p>.10]$.

Word association trials also tested knowledge of relations involving agent legs and movement orientation. The subjects averaged $71.1 \%(S D=30.5 \%)$ on tests of relations between nouns and agent legs, whereas they averaged $75.8 \%(S D=21.5 \%)$ on tests of relations between verbs and movement orientations. Both of these averages were significantly above chance $(p<.001)$, indicating that the subjects could differentiate nouns and verbs in terms of these two attributes and, thus, were not compelled by the experimental task to associate nouns and verbs with agent path and patient path.

\section{Mismatch Trials}

The results of the mismatch trials revealed that the subjects made a significantly greater percentage of choices consistent with the verb on trials in which agent path varied than on trials in which patient path varied $[t(31)=$ $2.08, p<.05]$. On trials in which agent path varied, $81.6 \%$ $(S D=22.4 \%)$ of the subjects' choices were consistent with the verb. This number was significantly greater than $50 \%(p<.001)$, indicating that the subjects associated agent path more strongly with verbs than with nouns. On trials in which patient path varied, $72.7 \%(S D=24.9 \%)$ of the subjects' choices were consistent with the verb. This number was also greater than $50 \%(p<.001)$, indicating that the subjects also associated patient path more strongly with verbs than with nouns.

The primary difference between mismatch trials and word association trials that tested knowledge of verbs was that the mismatch trials included a noun that was inconsistent with the accompanying verb. Thus, if nouns exerted any influence on performance in the mismatch trials, one would expect the percentage of choices consistent with the verb in the mismatch trials to be lower than the percentage of choices consistent with the verb (i.e., the percent correct) in the word association test trials. There was no significant difference between performance in the mismatch trials and performance in the word association trials for either patient path $[t(31)=0.91, p>.10]$ or agent path $[t(31)=0.55, p>.10]$. This suggests that nouns exerted no significant influence on mismatch trials.

\section{Discussion}

The results of Experiment 2 revealed that the subjects associated both the patient path and the agent path more strongly with verbs than with nouns. This finding is consistent with the division of labor hypothesis, because both agent path and patient path involve extrinsic motion. The subjects had difficulty associating nouns with paths, even though each agent always interacted with a particular patient and played a consistent role with respect to that patient (e.g., chaser). Moreover, both the agent and the patient were labeled in the accompanying transitive sentences, potentially highlighting the relation between the two characters.

The subjects associated nouns more strongly with the agent path than with the patient path. There are two possible explanations for this finding. One is that people have difficulty associating a noun with the motions of a character other than the one labeled by the noun. Although this explanation seems intuitively correct, the motion of this second character in part determined the relation of the agent to the patient in the present experiment. Such relations could be useful in determining the referent of a noun, even if it involves the motions of a different character (e.g., a cheetah is an animal that chases gazelles). An alternative explanation comes from the finding that verbs were also more strongly associated with the agent path than with the patient path. In particular, the agent 
path may simply have been more salient than the patient path, perhaps because it was carried by a larger character with an interesting manner of motion, or perhaps because it was labeled by the subject of the sentence. Thus, relations involving the agent path may have been easier to learn, regardless of the type of word involved.

Although the subjects had difficulty with relations between nouns and patient paths, they still performed significantly above chance on these relations, contrary to Experiment 1 . One may be tempted to conclude that the consistency of the role of the agent to the patient or the use of a transitive sentence facilitated the learning of relations between nouns and patient paths. An alternative explanation, however, is that the subjects simply relied on knowledge of relations between agent paths and patient paths, rather than knowledge associated with nouns. During learning, each patient path was always paired with a particular agent path. Thus, when the incorrect patient path was presented in a word association test trial, it was paired with an agent path that it had never accompanied during learning. Knowledge of which agent paths went with which patient paths may have allowed some subjects to choose the correct patient path, even if they had not learned its relation to the noun being tested. The subjects may also have relied to some extent on associations between agent paths and patient paths to perform successfully on tests of relations between nouns and agent paths. The subjects, however, performed significantly better on relations of nouns to agent paths than on those to patient paths. Thus, one can conclude that at least some subjects had more specific knowledge of relations of nouns to agent paths.

\section{EXPERIMENT 3}

The findings of Experiments 1 and 2 are consistent with a bias to associate extrinsic motion more strongly with verbs than with nouns. Experiments 3 and 4 were designed to investigate the association of an intrinsic motion attribute, movement orientation, with nouns and verbs. Movement orientation was found to be equally associated with nouns and verbs in both the word association and mismatch trials of Experiment 1. This finding differs from the findings of Kersten (1998), who found that subjects associated another intrinsic motion attribute, leg motion, more strongly with nouns than with verbs.

Findings from Kersten and Billman (1997), however, suggest an alternative explanation for the apparent equal association of movement orientation with nouns and verbs. This work revealed that subjects were able to learn relations between movement orientation and leg motion. In Experiment 1 as well, each leg motion always cooccurred with a particular movement orientation. If the subjects learned these relations between leg motion and movement orientation. this would have been sufficient for performance on tests of relations involving movement orientation. For example, when a subject who was assigned the schema in Figure 6 was tested on the relation of zeebee to moving backwards, both events in a test trial involved the leg motion consistent with zeebee. Only one event, however, involved a backward orientation, whereas the other involved a forward orientation. Thus, one test event involved a familiar pairing of leg motion and movement orientation, whereas the other involved an unfamiliar pairing. Knowledge of relations between leg motion and movement orientation would also have been sufficient on tests involving verbs. Thus, it is possible that the subjects relied on associations between leg motion and movement orientation on tests of both nouns and verbs. This would have resulted in equal performance on the two types of test.

Experiment 3 was designed to test the hypothesis that the subjects relied on associations between leg motion and movement orientation when they were tested on relations involving movement orientation. To this end, performance in a condition similar to that of Experiment 1 (the consistent leg motion condition) was compared with performance in a condition in which leg motion was not shown during testing (the obscured leg motion condition). Instead, the subjects in the obscured condition were instructed that the legs of the agent would remain motionless during testing and that they thus could not use this information to make their choices. If the subjects were relying on associations between leg motion and movement orientation in Experiment 1, the subjects in Experiment 3 would be expected to make fewer correct choices on tests of relations of movement orientations to both nouns and verbs when leg motion was obscured than when leg motion was visible during testing. These two conditions were also compared with a condition in which leg motion varied randomly throughout the experiment (the random leg motion condition). Thus, the subjects in the random condition never had the opportunity to associate values of leg motion and orientation. As a result, they were also expected to have difficulty on tests of relations involving movement orientation.

\section{Method}

\section{Subjects}

Sixty undergraduates at the Georgia Institute of Technology received course credit for participation in this experiment.

\section{Stimuli}

Learning events. The learning events in Experiment 3 were generated in the same way as those in Experiment 1, except that different attributes were correlated with the nouns and verbs that accompanied the events. In contrast to Experiment 1, only movement orientation was equally associated with nouns and verbs. Patien path no longer varied, but rather the patient remained stationary throughout each event. As in Experiment 1, agent path was the attribute that could be used to fully differentiate the four verbs, whereas agent legs differentiated the four nouns. In addition. for subjects in the consistent and obscured leg motion conditions, leg motion was perfectly correlated with agent legs, whereas leg motion was random in the random leg motion condition. 
Word association test events. For subjects in the obscured leg motion condition, these events varied from those of Experiment 1 in that the leg motion of the agent was not displayed. For subjects in the consistent and random leg motion conditions, these test events were identical to those of Experiment 1. In contrast to Experiment 1, nouns were only tested in relation to legs and to movement orientation, whereas verbs were only tested in relation to agent path and to movement orientation. Another difference from Experiment 1 was that each relation was tested eight times.

Mismatch test events. For subjects in the obscured leg motion condition, these events differed from those of Experiment 1 in that the leg motion of the agent was not displayed. For subjects in the consistent and random leg motion conditions, these events were identical to those of Experiment 1, except that only relations involving movement orientation were tested.

\section{Procedure}

The procedure of Experiment 3 was identical to that of Experiment 1 , except that there were 32 word association trials and 8 mismatch trials.

\section{Design}

The primary dependent measure was accuracy at choosing the correct events in the word association trials. One between-subjects independent variable was the relation of leg motion to movement orientation (consistent throughout, obscured at test, and random throughout). The second independent variable was the part of speech of the word accompanying each test trial (noun vs. verb). A second dependent measure was the percentage of choices that were consistent with the verb in the mismatch test trials. The independent variable for this dependent measure was the relation of leg motion to movement orientation.

\section{Results}

\section{Word Association Test Trials}

The results of the word association test trials are depicted in Figure 11. A 2 (part of speech, noun vs. verb) $\times$ 3 (leg motion, consistent vs. obscured vs. random) ANOVA was conducted on forced-choice accuracy. This analysis revealed a significant main effect of leg motion $[F(2,57)=$ $\left.5.56, M S_{\mathrm{e}}=629.52, p<.01\right]$. The main effect of part of

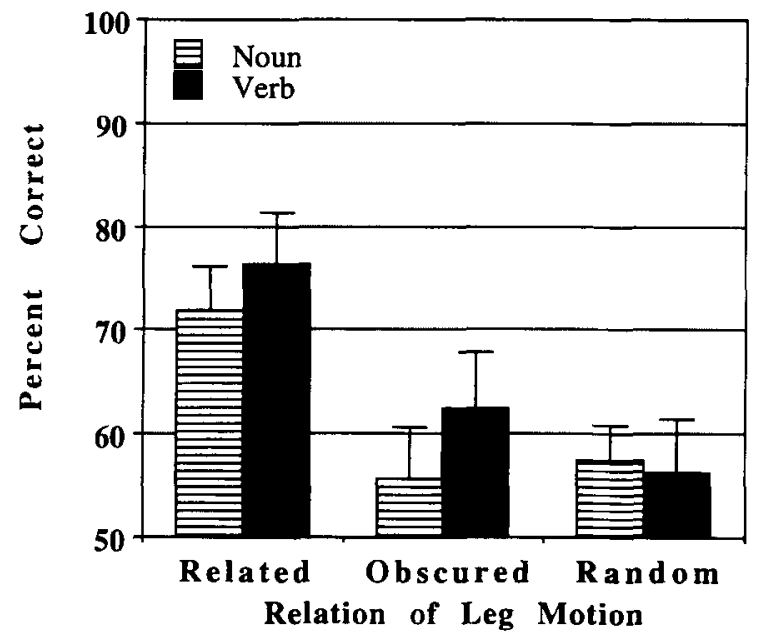

Figure 11. Results of the word association trials in Experiment 3. speech did not approach significance $[F(2,57)=1.18$, $\left.M S_{\mathrm{e}}=281.39, p>.10\right]$, nor did the interaction of part of speech with leg motion $[F(2,57)<1]$. Because there were no main effects or interactions involving part of speech, a combined measure of associations involving movement orientation was created by averaging each subject's measures of associations of nouns and verbs with movement orientation. These combined scores were then compared, to determine which conditions differed significantly from one another. A post hoc Fisher's least significant difference test revealed that the related leg motion condition scored significantly better than did both the obscured leg motion and the random leg motion conditions. There were no other significant differences between conditions. The subjects in the random leg motion condition failed to perform above chance on relations of either nouns or verbs to movement orientations ( $p>.10$ ), whereas the subjects in the obscured leg motion condition performed significantly above chance only on relations of verbs to movement orientations $(p<.05)$.

The subjects were also compared on performance in word association tests involving agent legs and agent path. The effects of leg motion on accuracy in tests of relations between nouns and agent legs were examined, using a one-way ANOVA. This analysis revealed that the difference between conditions approached significance $\left[F(2,57)=2.79, M S_{\mathrm{e}}=476.84, p<.07\right]$. The subjects in the consistent leg motion condition averaged $90.0 \%$ $(S D=17.0 \%)$ correct; the subjects in the obscured leg motion condition averaged $86.3 \%(S D=20.6 \%)$; and the subjects in the random leg motion condition averaged $74.4 \%(S D=26.7 \%)$. The same ANOVA was carried out on accuracy in tests of relations between verbs and agent paths. This analysis revealed no significant effect of leg motion $[F(2,57)<1]$. The subjects in the consistent leg motion condition averaged $87.5 \%(S D=20.3 \%)$; the subjects in the obscured leg motion condition averaged $91.3 \%(S D=12.9 \%)$; and the subjects in the random leg motion condition averaged $93.1 \%(S D=12.5 \%)$.

\section{Mismatch Trials}

The effects of leg motion on performance in the mismatch test events were analyzed, using a one-way ANOVA. This analysis revealed no significant differences between the three conditions $\left[F(2,57)=0.60, M S_{\mathrm{e}}=393.23, p>\right.$ .10]. Similar to the results of Experiment 1, the subjects in the consistent leg motion condition showed no preference that orientation be consistent with one part of speech over the other, with $55.0 \%(S D=29.9 \%)$ of choices being consistent with the verb. Similarly, the subjects in the random leg motion condition averaged $51.9 \%(S D=22.6 \%)$ of choices being consistent with the verb. The subjects in the obscured leg motion condition averaged $60.6 \%(S D=$ $23.7 \%$ ) of choices being consistent with the verb. The trend in this last condition approached significance $(p<$ $.06)$, with more choices being consistent with the verb than with the noun. 


\section{Discussion}

The results of Experiment 3 revealed that the subjects for whom leg motion was relevant and displayed during testing performed better on tests of relations involving movement orientation than did the subjects for whom leg motion was obscured during testing. This finding suggests that most subjects did not directly associate nouns and verbs with movement orientation. Instead, the successful performance of the subjects in Experiment 1 and of some subjects in Experiment 3 seems to have been primarily a result of their knowledge of which combinations of leg motion and movement orientation went together during learning. As a result, performance suffered when leg motion was not displayed during testing or when leg motion was unrelated to movement orientation throughout the experiment.

The subjects for whom leg motion was obscured during testing did reveal some direct knowledge of relations between verbs and movement orientation, however. Such associations between verbs and movement orientations help to explain the results of the mismatch trials. The subjects for whom leg motion was obscured during testing showed a trend toward making more choices consistent with the verb. Furthermore, the subjects for whom leg motion was relevant throughout showed no significant preference that movement orientation be consistent with one part of speech over the other. If these subjects had based their decisions entirely on the relation of movement orientation to leg motion, they would have made more choices consistent with the noun than with the verb. This is because the value of leg motion was always consistent with the noun during the mismatch trials for these subjects. Thus, knowledge of the relation of movement orientation to leg motion must have been offset by some knowledge of the relation of movement orientation to verbs. The trend toward more choices consistent with the verb in the obscured condition may represent the influence of associations between verbs and movement orientation in the absence of any influences in the opposite direction by leg motion.

\section{EXPERIMENT 4}

The results of Experiment 3 suggest that the relation of movement orientation to nouns was mediated by leg motion. The subjects revealed no direct knowledge of relations between nouns and movement orientations when leg motion was obscured at test time. Previous work by Kersten (1998), however, has demonstrated that subjects in this task do directly associate nouns with leg motions. Kersten used a method similar to the one employed in Experiment 3 to ensure that subjects had direct knowledge of relations between nouns and leg motions and could not rely on relations between the appearance of the legs and leg motion. In particular, the appearance of the legs was related to nouns during learning but was obscured during testing. Performance on relations between nouns and leg motions was quite comparable in this task with the performance of subjects for whom the legs were visible throughout.

Thus, the findings from Kersten (1998) showed that people associate nouns with leg motions, whereas the present findings reveal that people associate leg motions with movement orientations. It is interesting that the subjects did not make the further inferential step to determine the relation of nouns to movement orientations. Still, this combination of findings suggests that nouns, leg motions, and movement orientations are all associated together to form an object category. If this were the case, one would expect that manipulations that reduced the role of leg motion in object categories would also produce worse performance on relations involving movement orientation.

Kersten and Billman (1995) demonstrated the effects of one such manipulation. They found that subjects performed worse on relations between nouns and leg motions when the appearance of the legs carrying out those motions varied randomly throughout learning and only the appearance of the head was related to nouns. In contrast, relations of verbs to leg motions were unaffected by this manipulation. Thus, consistency in the appearance of object parts seems to selectively affect the role of the motions of those parts in object categories. Objeci category knowledge may also have formed the basis for subjects' successful performance on relations involving movement orientation in the present experiments. If so, one would expect that disrupting the relation of nouns to legs would affect not only knowledge of relations of nouns to leg motions but also knowledge of relations of leg motions to movement orientations. If object categories formed the sole basis for performance on relations involving movement orientation, one would expect disruptions in performance on tests of relations of movement orientations to verbs as well as to nouns. It is also possible, however, that the subjects relied on object categories only on tests of nouns and some other form of information (e.g., event categories) on tests of verbs. If so, the disruption of relations between nouns and legs would not be expected to affect performance on relations between movement orientations and verbs.

\section{Method}

Subjects

Fifty-six undergraduates at Indiana University participated in this experiment in partial fulfillment of introductory psychology course requirements.

\section{Stimuli}

Learning events. For half of the subjects, the learning events were identical to those of the consistent leg motion condition of Experiment 3 , with the appearance of the legs and leg motion differentiating the four nouns. For the other half, the appearance of the legs varied randomly, and only the head and leg motion of the agent could be used to differentiate the four nouns.

Word association test events. The subjects were tested eight times each on relations of nouns to leg motions, nouns to movement orientations, verbs to movement orientations, and verbs to agent paths.

Mismatch trials. These were identical to those of the obscured leg motion condition of Experiment 3. As in that experiment, the 
agents moved along their paths without moving their legs. This was done in order to examine the association of nouns and verbs with movement orientations, independently of leg motion.

\section{Procedure}

The procedure was identical to that of Experiment 3

\section{Design}

The primary dependent measure was accuracy at choosing the correct events in the word association test trials. One betweensubjects independent variable involved the static attribute that could be used to differentiate the four nouns (agent head vs. legs). A second independent variable was the part of speech of the word that accompanied each test trial (noun vs. verb). A second dependent measure was the percentage of choices that were consistent with the verb in the mismatch trials. The independent variable for this dependent measure was the attribute that differentiated nouns.

\section{Results}

\section{Word Association Test Trials}

The main prediction of this experiment was that the subjects would perform better on relations between nouns and movement orientations when they also learned the relation of nouns to leg motions. On the basis of the results of Kersten and Billman (1995), it was predicted that subjects who could use agent legs rather than heads to differentiate nouns would show better learning of relations between nouns and leg motions. Consistent with this prediction, the subjects were significantly more accurate on relations between nouns and leg motions when legs differentiated nouns $[t(54)=1.93, p<.05$, one-tailed $]$. The subjects averaged $77.7 \%(S D=19.9 \%)$ when legs differentiated nouns, as compared with an average of $65.6 \%$ $(S D=26.3 \%)$ when heads differentiated nouns. There was no significant effect of the choice of attribute to differentiate nouns on performance with relations between verbs and agent paths $[t(54)=0.42, p>.10]$. The subjects averaged $84.4 \%(S D=22.0 \%)$ when legs differentiated nouns, as compared with an average of $86.6 \%(S D=$

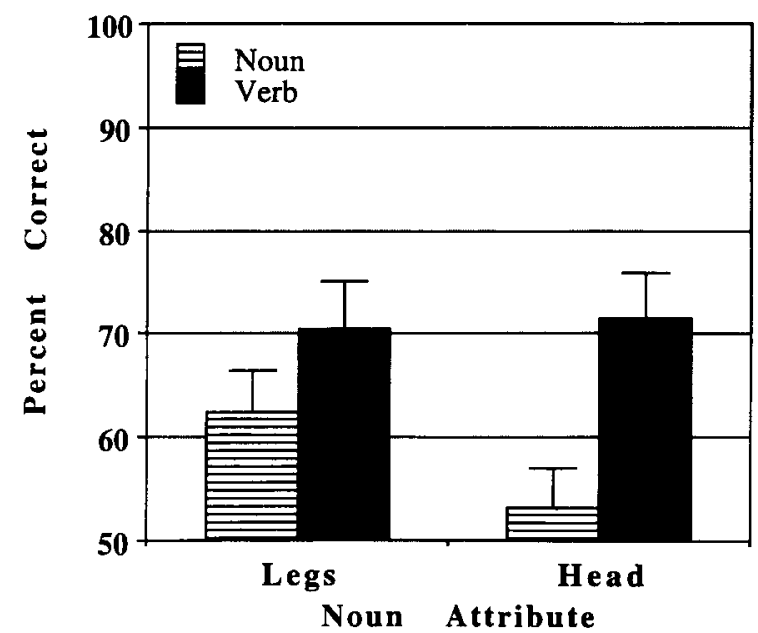

Figure 12. Results of the word association trials in Experiment 4 .
$18.0 \%$ ) when heads differentiated nouns. Thus, using heads to differentiate nouns did not lead to a general decrement in performance.

The results of word association trials testing knowledge of movement orientation are depicted in Figure 12. As predicted, the subjects who could use legs to differentiate nouns performed significantly better on tests of relations between nouns and movement orientations than did the subjects who could only use heads to differentiate nouns $[t(54)=1.70, p<.05$, one-tailed]. Moreover, across subjects in both conditions, there was a significant correlation between performance on relations of nouns to leg motions and performance on relations of nouns to movement orientations $[r(56)=.45, p<.001]$. Analysis of trials testing relations between verbs and movement orientations allows one to determine whether verbs exhibit the same effects as nouns. If this were the case, it would suggest that object categories formed the sole basis for the subjects' performance on relations involving movement orientation. Performance on relations between verbs and movement orientations, however, was not affected by the choice of attribute to differentiate nouns $[t(54)=0.14, p>.10]$. In addition, across subjects in both conditions, the correlation between performance on relations of nouns to leg motions and performance on relations of verbs to movement orientations was not significant $[r(56)=.08, p>.10]$.

Further evidence that object categories did not form the sole basis for performance on trials testing relations involving movement orientation comes from a 2 (part of speech, noun vs. verb) $\times 2$ (noun attribute, head vs. legs) ANOVA. This analysis revealed that subjects were significantly more accurate on tests involving verbs than on tests involving nouns $\left[F(1,54)=15.03, M S_{\mathrm{e}}=323.20\right.$, $p<.001]$. The subjects associated movement orientations more strongly with verbs than with nouns, not only when heads differentiated nouns $[t(27)=3.21, p<.01]$, but also when legs differentiated nouns $[t(27)=2.17, p<.05]$. The latter finding stands in contrast to the results of Experiments 1 and 3 . These experiments revealed equally strong associations of nouns and verbs with movement orientations when legs differentiated nouns. There was no significant main effect of noun attribute $[F(1,54)=0.73$, $\left.M S_{\mathrm{e}}=687.78, p>.10\right]$, nor did part of speech interact with noun attribute $\left[F(1,54)=2.28, M S_{\mathrm{e}}=323.20, p>.10\right]$.

\section{Mismatch Trials}

The results of the mismatch trials failed to reveal a significant effect of the choice of attribute to differentiate nouns $[t(54)=1.40, p>.10]$. The subjects who could use legs to differentiate nouns made an average of $55.8 \%$ $(S D=24.6 \%)$ of their choices consistent with the verb, not significantly different from $50 \%(p>.10)$. The subjects who could use only heads to differentiate nouns, however, made an average of $64.3 \%(S D=20.6 \%)$ of their choices consistent with the verb. The latter number was significantly greater than $50 \%(p<.01)$. Because leg 
motion was not displayed in the mismatch trials of this experiment, this finding provides further evidence for a direct association between verbs and movement orientations.

\section{Discussion}

Experiment 4 provides evidence that movement orientation plays a role in object categories. A manipulation previously shown to affect the association of leg motion with nouns was also demonstrated to affect performance on relations between movement orientations and nouns. Although Experiment 3 demonstrated that nouns are not directly associated with movement orientations, it did reveal knowledge of relations between leg motions and movement orientations. The present results suggest that this knowledge was associated with object categories.

Performance on relations between verbs and movement orientations was not affected by the choice of attribute to differentiate nouns. The results of Experiment 3 suggest that, in order to perform successfully on associations between verbs and movement orientations, the subjects relied primarily on knowledge of relations between leg motions and movement orientations. The results with tests of verbs in Experiment 4, in turn, suggest that these relations between leg motions and movement orientations were learned independently of the objects involved. This combination of findings suggests that relations between leg motions and movement orientations were associated not only with object categories but also with some independent knowledge base, perhaps event categories.

\section{GENERAL DISCUSSION}

These experiments provide evidence for different biases when learning nouns and verbs. The evidence most relevant to learning biases comes from an examination of how strongly the subjects associated a particular type of word with different kinds of information. This follows from the notion that people focus on different attributes of an event, depending on which type of word they are learning. Results with verbs were consistent with a bias to associate verbs with extrinsic motion, or the motion of one object with respect to another object. The subjects in Experiment 1 did better on tests of relations between verbs and an extrinsic motion attribute, patient path, than on those between verbs and an intrinsic motion attribute, movement orientation. This finding provides strong support for the extrinsic motion bias hypothesis, because patient path was carried by a character other than the one highlighted by the accompanying sentence, whereas movement orientation was carried by the highlighted character. Results with nouns were consistent with a bias to associate nouns with intrinsic motion, or motion with respect to an internal frame of reference. The subjects in Experiment 1 performed better on tests of relations between nouns and movement orientations than on tests of relations between nouns and patient paths.

The present research also provides evidence regarding a division of labor between nouns and verbs in the descrip- tion of motion. This evidence comes from an examination of the association of a particular attribute with nouns and verbs. In particular, do people rely on nouns more than on verbs to convey some types of information and on verbs more than on nouns to convey other types of information? This evidence is somewhat more complex. Consistent with the division of labor hypothesis, the subjects in Experiment 2 associated both agent path and patient path more strongly with verbs than with nouns. Findings with movement orientation, however, provide no evidence for a division of labor. In particular, the subjects in Experiment 1 performed equally well on relations of movement orientation to nouns and on those to verbs. Experiment 3, moreover, revealed that the subjects had not, in fact, directly associated nouns with movement orientations but rather that they were relying on associations between leg motions and movement orientations to perform successfully during testing. The results of Experiment 4 suggest that this knowledge of relations between leg motions and movement orientations was associated not only with object categories but also with verbs and event categories. Thus, the present results are consistent with the prediction that nouns and/or the object categories they label tend to be associated witl. intrinsic motion attributes, such as movement orientation. They are inconsistent, however, with a complete division of labor between object categories and verbs in the description of motion.

\section{The Role of Movement Orientation in Object Categories}

The present results extend previous findings showing a role for object orientation in object categories. In addition to playing a role in the interpretation of static spatial relations (Carlson-Radvansky \& Irwin, 1993), the present results indicate that an object's canonical orientation is taken into account when interpreting the motion of that object. As a result, if the objects in a particular category move in a consistent manner with respect to their canonical orientation, this movement orientation may be used when identifying members of that category.

Object categories in the present experiments apparently included knowledge of relations between the appearance of the legs of the agent, the motion of those legs, and the orientation of the agent as it moved, thus comprising a rich correlational structure (Rosch, Mervis, Gray, Johnson, \& Boyes-Braem, 1976). These properties may be associated together because they are all intrinsic properties of the agent. Nouns were not directly associated with movement orientations in these experiments. These associations were indirectly represented, however, through knowledge of relations between nouns and leg motions, along with object category knowledge about relations between leg motions and movement orientations.

The present results extend the findings of Kersten and Billman (1995) in showing that the association of intrinsic motions with object categories and nouns is dependent on the existence of consistent relations between those mo- 
tions and the static properties of objects. Just as Kersten and Billman (1995) found that people showed much weaker associations between nouns and leg motions when the appearance of the legs was random, the subjects in the present Experiment 4 performed much worse on tests of relations between nouns and movement orientations when the appearance of the legs was random. The subjects apparently failed to associate leg motions with object categories when the appearance of the legs was not also associated with those categories. As a result, object categories carried no information about relations between leg motions and movement orientations. This removed the subjects' only basis for performance on trials testing relations between nouns and movement orientations.

\section{The Association of Movement Orientation With Verbs}

Kersten (1998) proposed that object category knowledge also forms the basis for the association of intrinsic motion with verbs. People are biased to attend to extrinsic motion information when attempting to learn a verb, but this bias would fail when one is trying to learn verbs such as run, roll, and back (up), which describe different ways of achieving locomotion (i.e., intrinsic motion) but provide no information about the direction of motion with respect to other objects (i.e., extrinsic motion). Kersten proposed that, when extrinsic motion is found to be unrelated to verb meaning, learners look next to object knowledge to discover the meaning of a verb. In particular, learners aggregate over categories of objects that have been implicated in conjunction with the verb in question.

The results of Experiment 4 are inconsistent with the mechanism proposed by Kersten (1998), at least for associations involving movement orientation. When the appearance of the legs of the agent was random, the subjects failed to perform above chance on tests of relations between nouns and movement orientations, whereas they performed much better on tests involving verbs. If object category knowledge were the sole basis for the association of intrinsic motion with verbs and event categories, the subjects should have failed to perform any better with verbs than they did with nouns. The present results suggest that at least certain kinds of intrinsic motion can play a role in verb meanings and event categories, independently of their role in object categories. This may be the case because movement orientation involves the motion of an object as a whole and is, thus, similar to path. It remains possible that more prototypical intrinsic motions, such as leg motion, only become associated with verbs via object categories.

\section{Conclusions}

This research provides information regarding the types of motion that people tend to associate with nouns and verbs. Subjects associated verbs more strongly with an extrinsic motion attribute, patient path, than with an intrinsic motion attribute, the movement orientation of the agent. In contrast, subjects performed better on tests of relations between nouns and movement orientations than on tests of relations between nouns and patient paths. The subjects apparently relied on object category knowledge to perform successfully on tests of relations between nouns and movement orientations. The subjects relied equally on verbs and object categories to predict movement orientation, when both were available. This finding is interesting in that it violates the intuitive notion that verbs are primarily responsible for conveying motions.

Subjects often reported in postexperimental interviews that they attempted to relate the novel nouns and verbs they were hearing to nouns and verbs in English. For example, a subject may have considered the noun racha to be similar to roach and the verb morping to be similar to chase. As a result, the present biases may be considered to be a reflection of the types of information that the subjects had previously associated with real-world nouns and verbs. The different learning biases associated with nouns and verbs may reflect the different learning histories associated with these two classes of words (Kersten \& Smith, 1998). Thus, the present method offers not only a means of investigating how people learn novel concepts but also a picture of the types of information that are represented in established concepts.

\section{REFERENCES}

Behrmann, M., \& Moscovitch, M. (1994). Object-centered neglect in patients with unilateral neglect: Effects of left-right coordinates of objects. Journal of Cognitive Neuroscience, 6, 1-16.

BisiaCH, E., \& VALLAR, G. (1988). Hemineglect in humans. In F. Boller \& J. Grafman (Eds.), Handbook of neuropsychology (Vol. 1, pp. 195222). Amsterdam: Elsevier.

BROWN, R. (1957). Linguistic determinism and the part of speech. Journal of Abnormal \& Social Psychology, 55, 1-5

Caramazza, A., \& Hillis, A. E. (1990). Spatial representation of words in the brain implied by studies of a unilateral neglect patient. Nature, 346, 267-269.

Carlson-Radvansky, L. A., \& IRWin, D. E. (1993). Frames of reference in vision and language: Where is above? Cognition, 46, 223-244.

Driver, J., \& Halligan, P. W. (1991). Can visual neglect operate in object-centred co-ordinates? An affirmative single-case study. Cognitive Neuropsychology, 8, 475-496.

Gleitman, L. R., Gleitman, H., Landau, B., \& Wanner, E. (1987). Where learning begins: Initial representations for language learning. In F. Newmeyer (Ed.), The Cambridge linguistic survey (Vol. 3, pp. 150-193). Cambridge: Cambridge University Press.

JACKENDOFF, R. (1987). On beyond zebra: The relation of linguistic and visual information. Cognition, 26, 115-122.

Kersten, A. W. (1998). A division of labor between nouns and verbs in the representation of motion. Journal of Experimental Psychology: General, 127, 34-54

Kersten, A. W., \& Billman, D. (1995). The roles of motion and moving parts in noun and verb meanings. In J. D. Moore \& J. F. Lehman (Eds.), Proceedings of the seventeenth annual conference of the Cognitive Science Society (pp. 625-630). Hillsdale, NJ: Erlbaum.

KeRSTen, A. W., \& BilLman, D. (1997). Event category learning. Journal of Experimental Psychology: Learning. Memory, \& Cognition 23, 638-658. 
Kersten, A. W., \& Smith, L. B. (1998). Attention to novel objects dupring verh learning. Manuscript submitted for publication.

Maratsos, M., \& Chalkley, M. A. (1980). The internal language of children's syntax: The ontogenesis and representation of syntactic categories. In K. Nelson (Ed.), Children's language (Vol. 2, pp. 127 214). New York: Gardner.

NaGy, W., \& Gentner. D. (1990). Semantic constraints on lexical categories. Language \& Cognitive Processes, 5, 169-201.

Naigles, L. (1990). Children use syntax to learn verb meanings. Journal of Child Language, 17, 357-374.

NeLson, K. (1983). The derivation of concepts and categories from event representations. In E. Scholnick (Ed.), New trends in conceptual rep- resentation: Challenges to Piaget's theory (pp. 129-150). Hillsdale, NJ: Erlbaum

Rosch, E. H., Mervis, C. B., Gray, W. D., Johnson, D. M., \& BoyesBraem, P. (1976). Basic objects in natural categories. Cognitive Psychology, 8, 382-439

TALMY, L. (1983). How language structures space, In H. Pick \& L. Acredolo (Eds.), Spatial orientation: Theory, research, and application (pp. 225-282). New York: Plenum.

(Manuscript received July 22, 1996; revision accepted for publication May $15,1997$. 